\title{
Design of Wideband Inverted Sinc Shaped Monopole Antenna for Wireless Applications
}

\author{
Ritesh Kumar Badhai and Nisha Gupta \\ Electronics and Communication Engineering,Birla Institute of Technology Patna \\ Campus, Patna, India \\ Electronics and Communication Engineering, Birla Institute of Technology, \\ Mesra, Ranchi, India \\ riteshkbadhai@bitmesra.ac.in,ngupta@bitmesra.ac.in
}

\begin{abstract}
A novel wideband inverted Sinc shaped printed monopole antenna is proposed for wireless devices. The proposed configuration not only realizes the monopole antenna in compact form but also depicts good radiation characteristics for wideband operations. A detailed parametric study has been carried out for different size of the ground plane, feed gap and Sinc strip length. By selecting the proper dimensions of ground plane, feed gap and Sinc strip length, the broadband operation for the proposed design can be achieved and the obtained impedance bandwidth covers the 2855-5081 $\mathrm{MHz}$ band. The proposed monopole antenna provides a wide frequency band of about $56 \%$ for wireless operation. The peak gain and efficiency of the proposed antenna are $4.05 \mathrm{dBi}$ and $92.20 \%$ respectively. Finally a prototype model is developed for the most effective configuration and the simulated results are validated experimentally. It is seen that the proposed antenna occupies small area and is suitable for operation at radio navigation, space research, amateur radio, aviation, public safety mobile, fixed mobile and fixed satellite communication frequencies.
\end{abstract}

Keywords: Monopole antenna, printed antenna, sinc antenna, wideband antenna

\section{Introduction}

Modern wireless devices demand antennas having features such as light weight, planar conformal construction, low cost, easy to fabricate and integrate with RF devices, etc. At the same time it must be able to accommodate several bands of operation. Monopole antennas [1] are most suited for such applications as it fulfills most of the requirements of modern wireless devices and it shows broadband characteristics at operating frequencies.

The increasing use of mobile communication systems has stimulated the interest in the wideband frequency design of monopole antennas for application in wireless communication systems. Many printed strip monopole antennas have been designed for the wireless applications. Important structures among them are printed strip antenna [2], wideband printed monopole [3,4], monopole antenna with dual strip [5], Y-shaped antenna [6,7], Trident-shaped [8], T-shaped [9] monopole with L shaped sleeve, V-shaped antenna [10], S-shaped folded strip monopole antenna [11], a sinusoidal shaped antenna [12-14] etc. Most of these antennas have complex geometrical shapes and involve difficult design steps. Several techniques for improving the wideband performance of antenna have been reported in litrature such as, use of modifide ground structures [15], use of shorting pins [16-17], and use of dielectric slab [18]. However these techniques are required modification in ground structure or fed by probe which involves difficult design steps.

In the present work, a novel monopole antenna is proposed where the shape of the monopole is derived from Sinc function, in order to realize a compact and simple antenna 
configuration. The shape of the monopole configuration resembles to an inverted and truncated Sinc strip. By changing the Sinc geometrical parameters, the antenna can be made to operate at the desired band of frequencies. The Sinc shaped microstrip configuration was first proposed by Yang et. al., [19] and then Gupta et. al., [20] also used a similar configuration to realize multifrequency antenna. However, in both these configurations the shape of the antenna was only Sinc alike and not exactly derived from the Sinc function. In the present work, a truncated and inverted Sinc shaped printed monopole antenna geometry is obtained directly from the Sinc function. The monopole geometry under consideration is a truncated Sinc giving rise to one of the compact monopole configurations. The simulation is performed for several truncated Sinc shaped monopole geometries printed on a low cost glass epoxy substrate. Finally a prototype is developed and the results are compared with the simulation results.

The proposed antennas are useful for several wireless applications such as radio navigation (2900-3100 MHz), space research (3100-3300 MHz), amateur radio (3300$3500 \mathrm{MHz}$ ), aviation (4200-4500 MHz), public safety mobile (4800-4900 MHz), fixed mobile $(4438-4650 \mathrm{MHz})$ and fixed satellite space to earth $(4438-4650 \mathrm{MHz})$ communication

\section{Antenna Design}

The antenna designers always explore ways to realize compact antenna configuration that can be designed easily; impedance matched easily and yet be capable of covering more than one band of frequencies. One such antenna is an inverted Sinc shaped monopole antenna. First of all, larger length can be accommodated in smaller area thus realizing a compact structure. Secondly the shape can be generated by a simple mathematical Sinc function. Thirdly, single, dual or multi band operation can be realized, simply by selecting a specific length of the Sinc monopole or ground plane parameters. The impedance matching is not an issue at all for all possible design parameters. Unlike the meander line strip where there is more opposition of current vectors in the parallel section of strips leading to diminishing self-inductance, in the proposed Sinc strip configuration, the current vectors reinforce along the total strip length thus adding to more self-inductance. This in turn is more effective in realizing a compact antenna configuration. The Sinc shaped antenna of any dimension along the length or width can be visualized as one of the Sinc functions $y=a\{\sin (b \pi x) /(b \pi x)\}$, printed in the form of a strip on a substrate. The antenna dimension can be increased or decreased along the length as much needed, by taking the suitable value of the coefficient ' $b$ ' of the argument $\mathrm{x}$; and along the height by taking appropriate factor ' $a$ ' of the function. In the proposed truncated Sinc shaped monopole configuration, the constants are considered as $a=1$ and $b=2$. Actually, the Sinc function extends up to infinity, therefore in practice, the Sinc antenna refers to a 'truncated' Sinc function, the range of the function determining the length of the monopole strip.

The Sinc function with truncated markers between $a^{\prime}$ and $a$ " is shown in Fig. 1. The specific function $\operatorname{Sinc}(2 x)$ is considered. The geometry of the Sinc is obtained from the Sinc function using MATLAB and then imported to the IE3D, a full-wave method of moments $(\mathrm{MoM})$ based simulation software package from Zeland. The desired strip thickness is then set by setting the thickness of the line. The vertex of Sinc function imported from MATLAB is then multiplied with the desired factor in order to operate the antenna at specific band. In the proposed antenna the imported geometry is multiplied by a factor of 10. The radiation characteristic of the Sinc shaped antenna is examined with respect to several design parameters. The parametric analysis of the Sinc strip is performed both on finite and infinite substrate size, and for different set of design parameters. The monopole strip width is maintained same as the width of the $50 \Omega$ microstrip line and simple microstrip feeding mechanism is used for feeding the inverted Sinc monopole. The proposed inverted Sinc shaped configuration is shown in the Figure 2 
The various parameters are selected as dielectric constant $\left(\varepsilon_{r}\right)=4.4$, loss tangent $(\tan \delta)$ $=0.018$, dielectric thickness $(h)=1.57 \mathrm{~mm}$, width of the $50 \Omega$ microstrip line equal to width of the Sinc strip $(W s)=3.0 \mathrm{~mm}$, feed gap $(g)=3 \mathrm{~mm}$, the ground plane size $W g=$ $30 \mathrm{~mm}, L g=20 \mathrm{~mm}$, antenna size $L=40 \mathrm{~mm}, W=W g=30 \mathrm{~mm}$ and Sinc parameters: Sinc strip length $(L p)=10 \mathrm{~mm}$, Sinc strip width $(W p)=3 \mathrm{~mm}$ Sinc strip height $(L e)=10$ $\mathrm{mm}$. for $\operatorname{Sinc}(2 x)$ configuration.

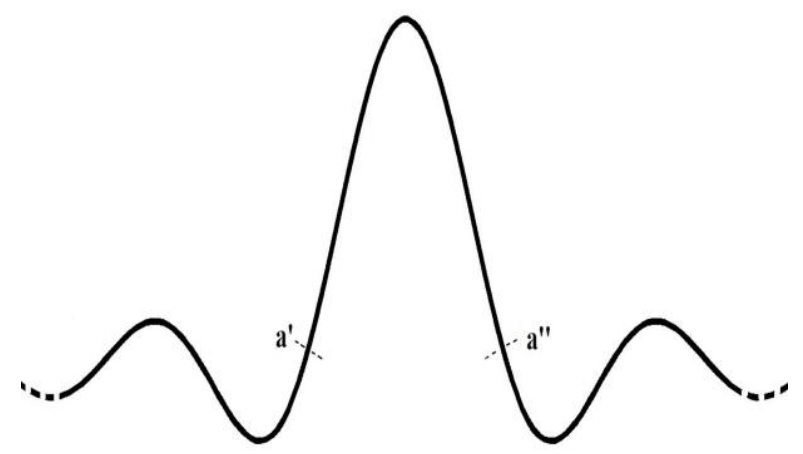

Figure 1. Sinc Function with the Truncated Section between a' and a".

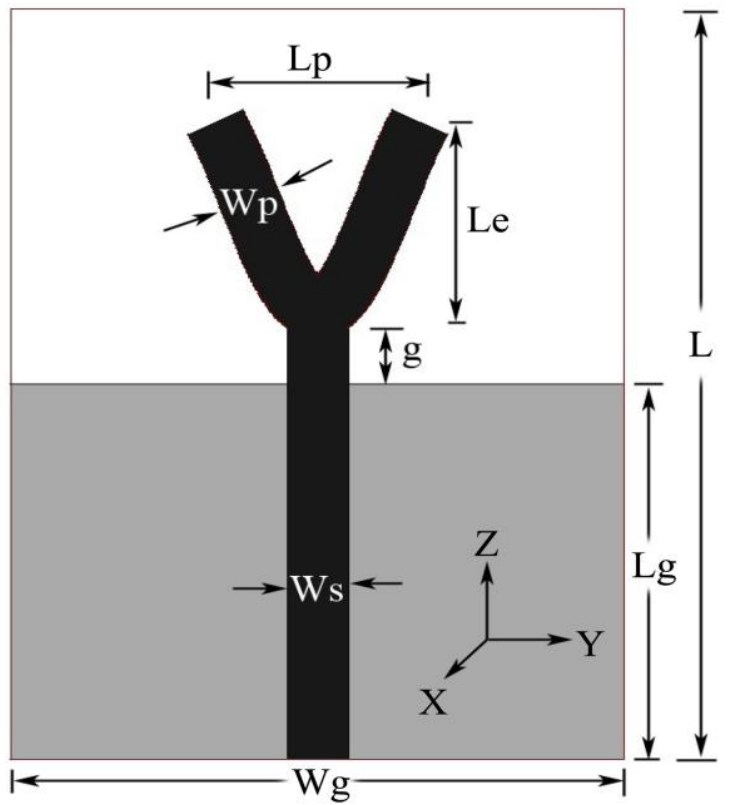

Figure 2. Inverted Sinc Shaped Monopole Antenna Configuration.

\section{Parametric Study}

\section{A. Effect of the Ground Plane}

The ground plane dimensions $(L g, W g)$ are very important parameters in the design of a monopole antenna because of the strong dependence of gain, bandwidth and antenna efficiency on ground plane size. Simulations are performed with various ground plane parameters and the effects of the ground plane size on resonant frequencies are studied.

1) Effect of Ground Plane Length ( $L g)$ : As shown in Figure 3, the ground plane length $(L g)$ affects the resonant frequency of inverted Sinc shaped monopole configuration. By increasing the length $(L g)$, a decrease in resonance frequencies is evident. The bandwidth of antenna first increases and then decreases with increasing $L g$ as shown in Figure 4. 
2) Effect of Ground Plane Width $(W g)$ : The effect of ground plane width on antenna characteristics is shown in Figure 5. As evident from the figure, the ground plane width $(W g)$ does not affect the resonant frequency; however it has an adequate impact on the bandwidth. The bandwidth of antenna linearly decreases with increasing $W g$ for the constant length of ground $(L g)$ as shown in Figure 6.

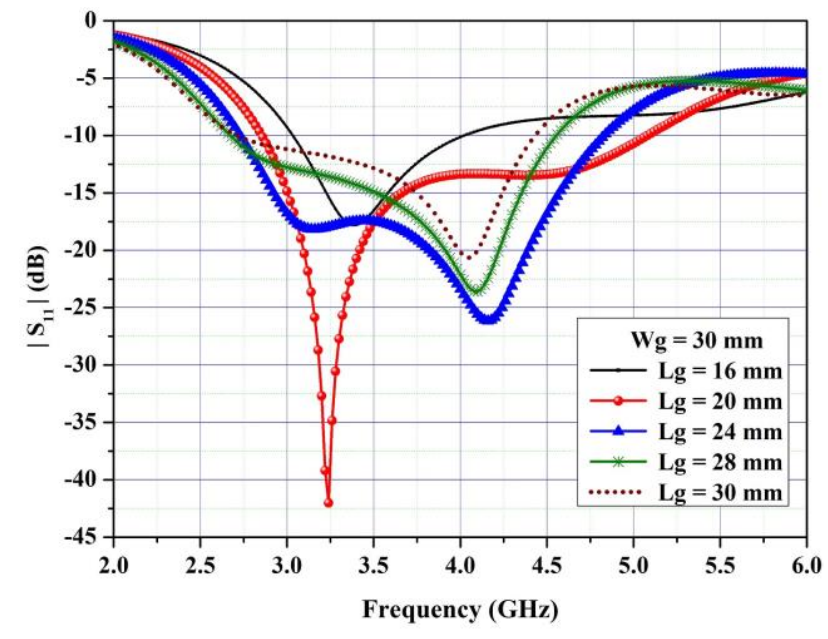

Figure 3. Effect of Ground Plane Dimensions on Reflection Coefficient $\left(\mid S_{11}\right)$ with Respect to $L g$.

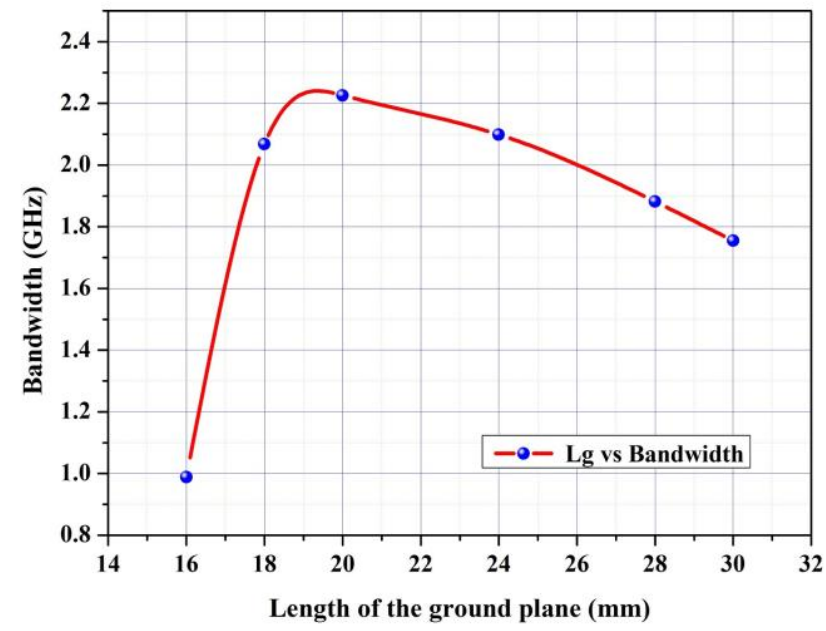

Figure 4. Effect of Ground Plane Length on Bandwidth.

For the maximum bandwidth $W g=30 \mathrm{~mm}$ and $L g=20 \mathrm{~mm}$ are selected which also provides good impedance matching for selected Sinc parameters. 


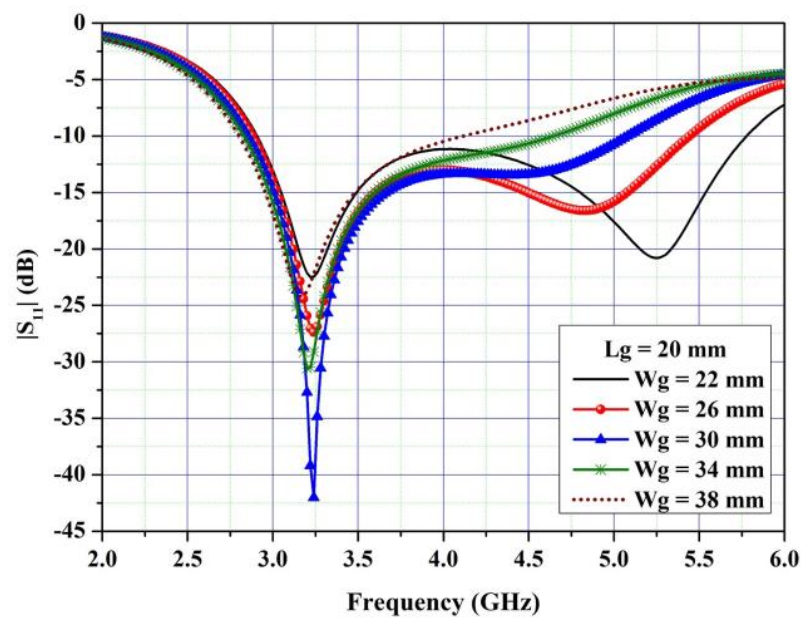

Figure 5. Effect of Ground Plane Dimensions on Reflection Coefficient $\left(/ S_{11}\right)$ with Respect to $\mathbf{W g}$.

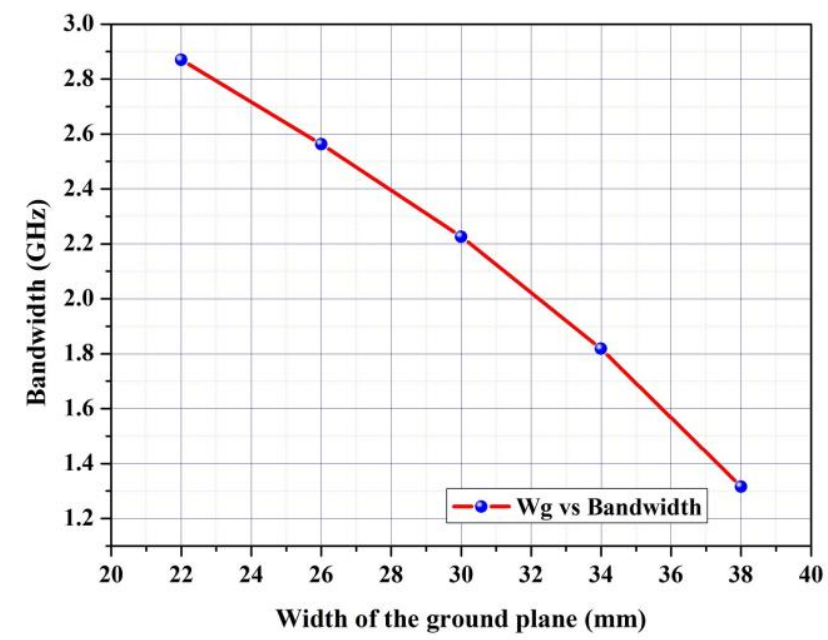

Figure 6. Effect of Ground Plane Width on Bandwidth.

\section{A. $\quad$ Effect of the Feed Gap}

Next the effect of the feed gap on the characteristics of the antenna is examined. The effect of feed gap on resonant frequency and reflection coefficient is shown in Figure 7. For the different feed gaps $(g)$ between the Sinc strip and the ground plane, it is seen that the resonance frequency reduces with increasing feed gap $(g)$, while bandwidth and impedance matching first increase and then decrease with increasing feed gap $(g)$. 


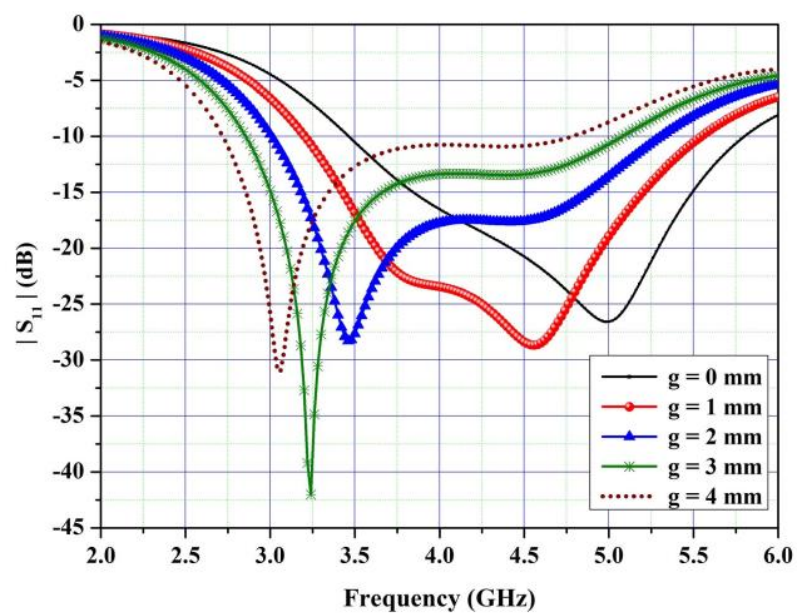

Figure 7. Effect of Feed Gap on Reflection Coefficient $\left(/ S_{11}\right)$.

\section{B. Effect of Sinc Strip Length}

To optimize the performance of the inverted Sinc shaped monopole antenna varius strip lengths between $10 \mathrm{~mm}$ to $30 \mathrm{~mm}$ are considered. The effects of the Sinc strip length $L p$ on antenna performance are shown in Figure 8. As evident from the Figure 8 the resonant frequency and bandwidth of the antenna reduces with increasing sinc strip length $L p$. The Sinc strip length $L p=16$ to $30 \mathrm{~mm}$ provides dual band operation while the second resonance frequency $f_{2}$ for $L p=10 \mathrm{~mm}$ shift towards the higher frequency. From Table I it is seen that the first resonance frequency is varies from 3.23 to $2.28 \mathrm{GHz}$, and second resonance frequency varies from $7.93 \mathrm{GHz}$ to $6.70 \mathrm{GHz}$ for Sinc strip length $L p=$ $10 \mathrm{~mm}$ to $30 \mathrm{~mm}$. It is found that the maximum bandwidth is obtained when the Sinc strip length $L p=10 \mathrm{~mm}$ and it also provides good impedance matching.

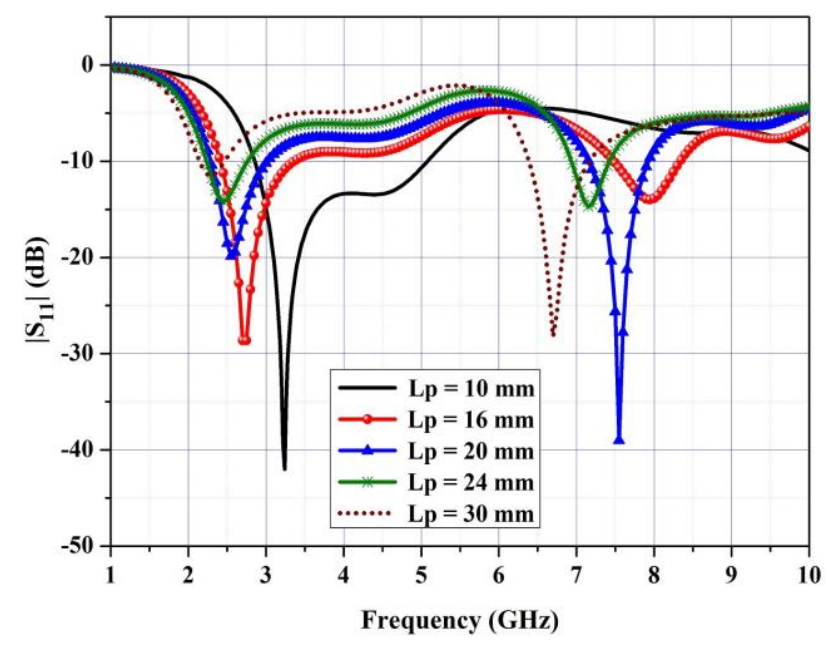

Figure 8. Effect of Sinc Strip Length on Reflection Coefficient $\left(/ S_{11}\right)$.

Table 1. Effect of Sinc Strip Length

\begin{tabular}{ccccccc}
\hline $\begin{array}{c}\text { Sinc strip } \\
\text { length } \\
(\mathbf{m m})\end{array}$ & $f_{1}$ & $\left|\mathbf{S}_{\mathbf{1 1}}\right|$ & $\mathbf{B W}$ & $f_{2}$ & $\left|\mathbf{S}_{\mathbf{1 1}}\right|$ & $\mathbf{B W}$ \\
\hline 10 & $(\mathbf{G H z})$ & $(\mathbf{d B})$ & $(\mathbf{M H z})$ & $(\mathbf{G H z})$ & $(\mathbf{d B})$ & $(\mathbf{M H z})$ \\
\hline
\end{tabular}




\begin{tabular}{llccccc}
\hline 16 & 2.78 & - & 953 & 7.93 & -13.96 & 857 \\
20 & 2.55 & $\begin{array}{c}28.63 \\
-\end{array}$ & 732 & 7.55 & -37.47 & 775 \\
24 & 2.45 & $\begin{array}{c}19.81 \\
-\end{array}$ & 530 & 7.15 & -14.65 & 491 \\
30 & 2.28 & $\begin{array}{c}14.18 \\
-\end{array}$ & 322 & 6.70 & -27.78 & 781 \\
\hline
\end{tabular}

\section{Experimental Results}

Finally, the prototype model is developed for truncated inverted $\operatorname{Sinc}(2 x)$ configuration as shown in Figure 9, and the experimental results of the reflection coefficient $\left(\left|S_{I I}\right|\right)$ of the proposed antenna is obtained using PNA series of Vector Network Analyzer. The current distribution of inverted $\operatorname{Sinc}(2 x)$ antenna is shown in Figure 10 at $3.30 \mathrm{GHz}$. In the prototype model developed, the length of the Sinc strip is considered as $L p=10 \mathrm{~mm}$ for proposed inverted Sinc shaped configuration. A comparison of the experimental and simulation results are shown in Figure 11. A good agreement between the results are evident. The simulated and measured normalized radiation patterns of the inverted Sinc shaped monopole antenna at $3.30 \mathrm{GHz}$ are shown in Figure 12 and it can be seen that the radiation pattern in X-Y plane are nearly omnidirectional pattern. The antenna also shows a good cross polarization, gain and efficiency characteristics for the proposed monopole configuration. The gain of the antenna in the proposed $(2855-5081 \mathrm{MHz})$ band is presented in Figure 13. The peak gain of the proposed antenna is $4.05 \mathrm{dBi}$ and average gain is $3.25 \mathrm{dBi}$. The efficiency of the antenna in the proposed $(2855-5081 \mathrm{MHz})$ band is presented in Figure 14 and the maximum antenna efficiency of the proposed antenna is $92.20 \%$ obtain.

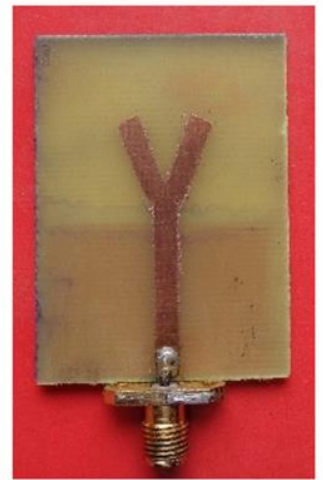

Top view

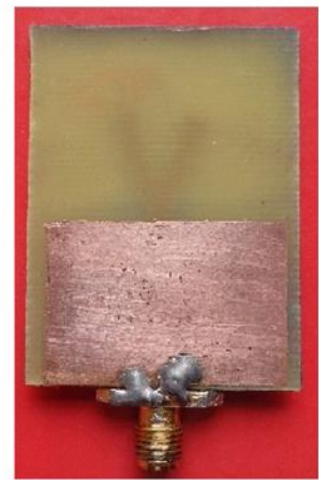

Bottom view

Figure 9. Fabricated Inverted $\operatorname{Sinc}(2 x)$ Monopole Antenna. 


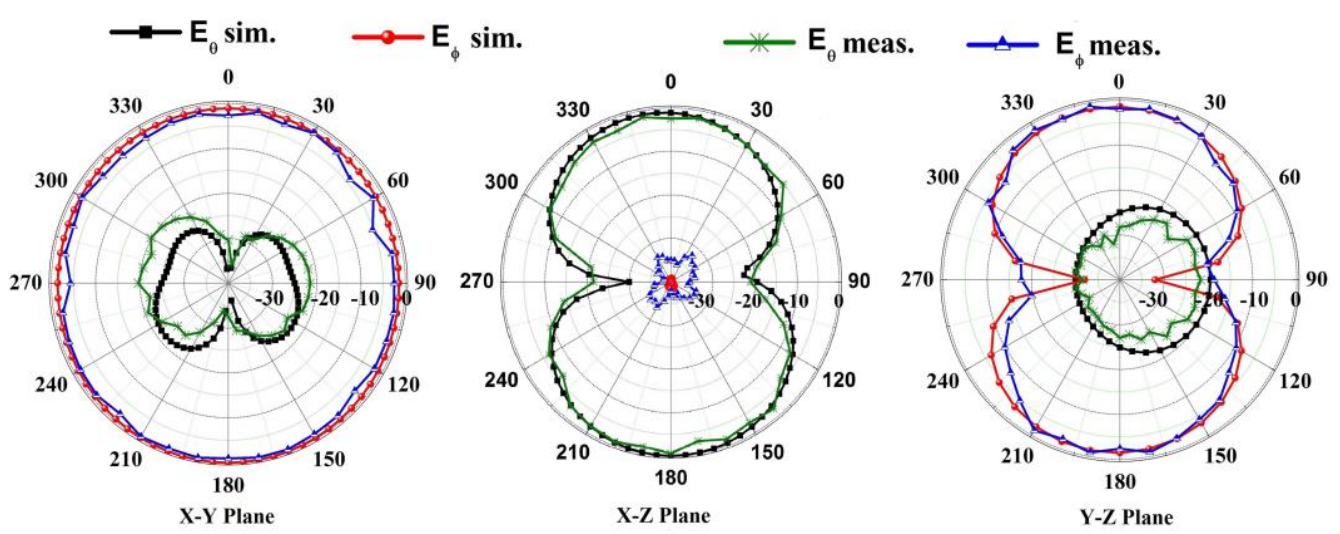

Figure 12. Normalized Radiation Patterns of the Inverted Sinc Shaped Monopole Antenna at $3.30 \mathrm{GHz}$.

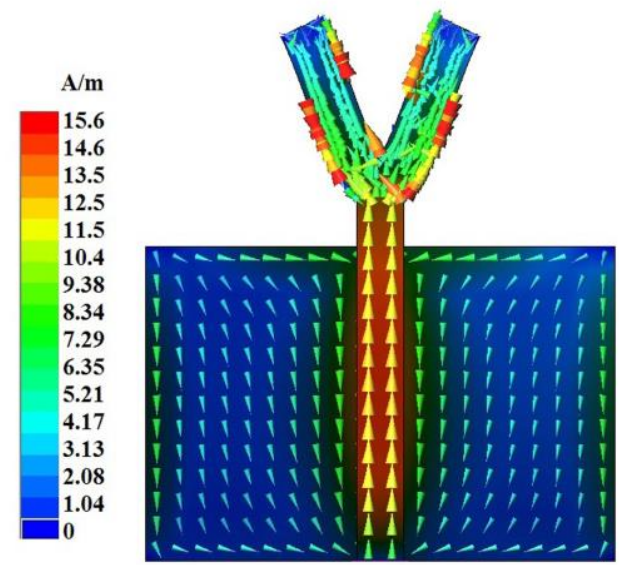

Figure 10. The Current Distribution of Inverted Sinc Shaped Monopole Antenna at $3.30 \mathrm{GHz}$.

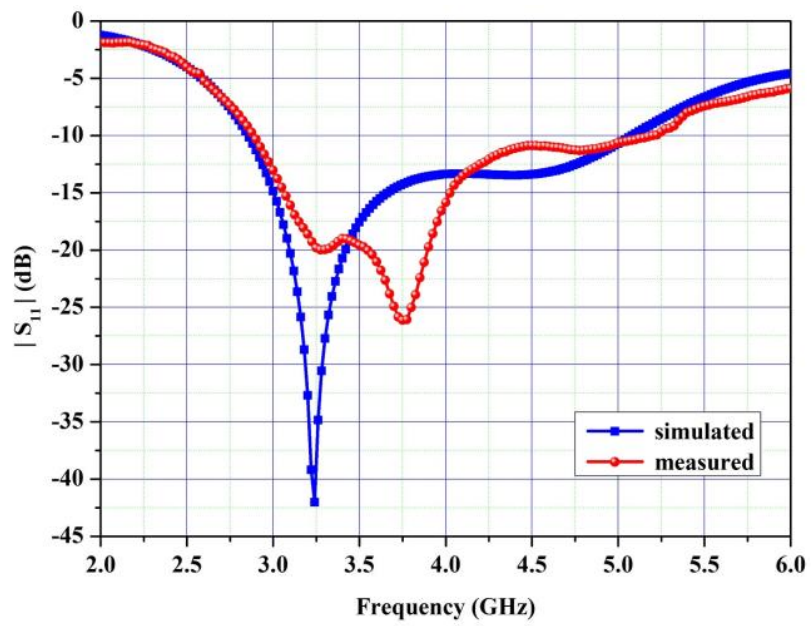

Figure 11. Simulated and Measured Reflection Coefficient $\left(/ S_{11}\right)$ of the Inverted Sinc Shaped Monopole Antenna. 


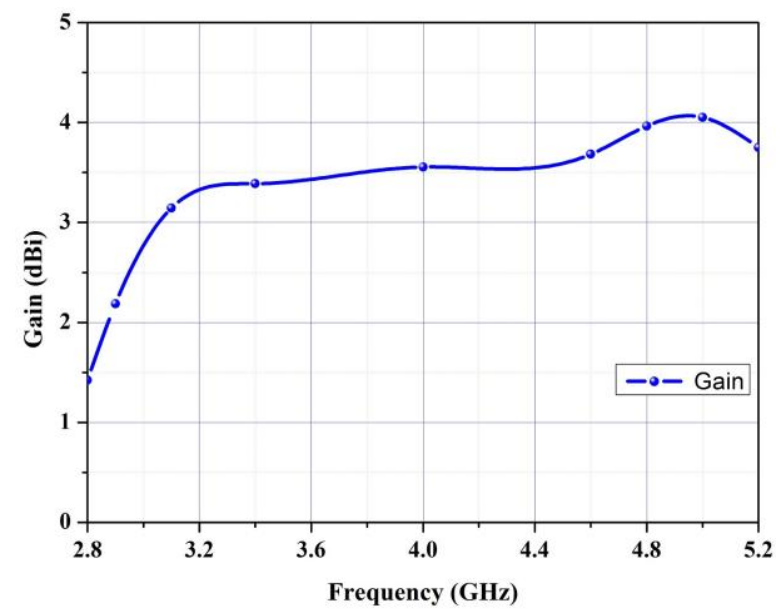

Figure 13. Peak Gain of the Inverted Sinc Shaped Monopole Antenna.

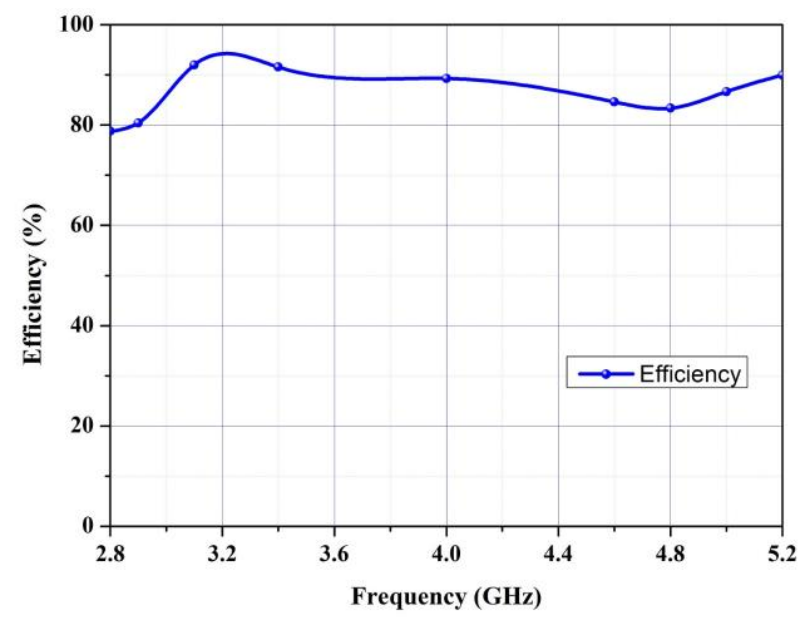

Figure 14. Efficiency of the Inverted Sinc Shaped Monopole Antenna.

\section{Conclusion}

A new configuration of monopole antenna is proposed for wireless communication. It has been shown that the inverted Sinc shaped monopole antenna is a very good candidate for wideband operation used in wireless communication. A detailed parametric study of the proposed antenna indicates that the desired band of operation can be obtained simply by setting the Sinc monopole design parameters without changing the shape of proposed configuration. The proposed antenna configurations occupy an area of $30 \times 40 \mathrm{~mm}^{2}$. The antenna is found to be useful for several wireless applications such as radio navigation, space research, amateur radio, satellite communication, aviation and public safety mobile.

\section{References}

[1] M. E. Bialkowski, A. R. Razali, and A. Boldaji, "Design of an ultrawideband monopole antenna for portable radio transceiver," Antennas and Wireless Propagation Letters, IEEE, vol. 9, pp. 554-557, 2010.

[2] M. J. Ammann and M. John, "Optimum design of the printed strip monopole," IEEE Magzine Antennas Propag., vol. 47, no. 6, pp. 59-61, Dec 2005.

[3] M. N. Suma, P. C. Bybi, and P. Mohanan, "A wideband printed monopole antenna for 2.4-GHz WLAN applications," Microw. Opt. Technol. Lett, vol. 48, pp. 871-873, 2006.

[4] N. Agrawall, G. Kumar, and K. P. Ray, "Wide-band planar monopole antennas," Antennas and Propagation, IEEE Transactions on, vol. 46, no. 2, pp. 294-295, 1998. 
[5] J. H. Yoon and G. S. Kil, "Compact monopole antenna with two strips and a rectangular-slit ground plane for dual-band WLAN/WiMAX applications," Microwave and Optical Technology Letters, vol. 54, no. 7, pp. 1559-1566, 2012.

[6] H. M. Hsiao, J. H. Lu, and J. W. Wu, "Y-shaped monopole antenna with dual-broadband operation for WLAN," Microw. Opt. Technol. Lett, vol. 48, no. 8, 2006.

[7] J. S. Mandeep and T. C. How, "Design of a dual monopole antenna with wideband frequency," Progress In Electromagnetics Research C, vol. 3, pp. 119-128, 2008.

[8] Wang, Z. H. Yan, P. Xu, J. B. Jiang, and B. Li, "Trident-shaped dual-band CPW-fed monopole antenna for PCS/WLAN applications," Electronics Letters, vol. 47, no. 4, pp. 231-232, 2011.

[9] J. W. Wu and Y. D. Wang, "T-shaped monopole antenna with shorted L-shaped strip-sleeves for WLAN 2.4/5.8-GHz operation," Microw. Opt. Technol. Lett, vol. 46, no. 1, pp. 65-69, July 2005.

[10] P. C. Ooi and K. Selvan, "A novel V-shaped CPW-fed printed antenna for WLAN/WiMAX applications," in Microwave Conference, 2009. APMC 2009. Asia Pacific, 2009, pp. 1799-1801.

[11] W. Liu, W.-R. Chen, and C.-M. Wu, "Printed double S-shaped monopole antenna for wideband and multi-band operation of wireless communications," IEE, Proceedings on Microwaves, Antennas and Propagation, vol. 151, no. 6, pp. 473-476, 2004.

[12] G. Kakoyiannis and P. Constantinou, "Radiation properties and ground-dependent response of compact printed sinusoidal antennas and arrays," Microwaves, Antennas Propagation, IET, vol. 4, no. 5, pp. 629$642,2010$.

[13] G. Kakoyiannis, P. Gika, and P. Constantinou, "Small printed sinusoidal antennas: A simple design guide for smooth meander-line structures with augmented bandwidth," in Antenna Technology, 2009. iWAT 2009. IEEE International Workshop on, 2009, pp. 1-4.

[14] G. Kakoyiannis and P. Constantinou, "Compact WSN antennas of analytic geometry based on chebyshev polynomials," in Antennas and Propagation Conference (LAPC), 2012 Loughborough, 2012, pp. 1-6.

[15] J. Y. Jan and T.-M. Kuo, "CPW-fed wideband planar monopole antenna for operations in DCS, PCS, 3G, and bluetooth bands," Electronics Letters, vol. 41, no. 18, pp. 991-993, 2005.

[16] Lee, P. Hall, and P. Gardner, "Compact wideband planar monopole antenna," Electronics Letters, vol. 35, no. 25, pp. 2157-2158, 1999.

[17] GS Tomar, Vivek Kushwah, Sarita Bhadoria, "Artificial Neural Network Design of Stub Microstrip Band-pass Filters", Inderscience International Journal of Ultra Wideband Communications and Systems, Vol.3, No.1, pp-38-49, 2014.

[18] M. Ammann and Z.-N. Chen, "A wide-band shorted planar monopole with bevel," Antennas and Propagation, IEEE Transactions on, vol. 51, no. 4, pp. 901-903, 2003.

[19] Z. Chen, "Broadband planar monopole antenna," Microwaves, Antennas and Propagation, IEE Proceedings, vol. 147, no. 6, pp. 526-528, 2000.

[20] Sweety Jain, Pankaj Singh Tomar, G.S. Tomar, "Design \& Analysis of Proximity Fed Circular Disk Patch Antenna", International Journal of Emerging Technology and Advanced Engineering Volume 2, Issue 10, pp 126-131, October 2012.

[21] H. Y. D. Yang, J. A. Castaneda, and F. D. Flaviis, "A novel printed sinc antenna for wireless communications,” IEEE Antenna and Propag.Society Int. Symp., vol. 4, pp. 48-51, 2002.

[22] N. Gupta, M. Mishra, S. C. Kotha, and Y. Kiran, "Multifrequency characteristics of sinc shaped microstrip patch antenna," Microw. Opt. Technol. Lett, vol. 49, no. 7, pp. 1673-1675, 2007

\section{Authors}

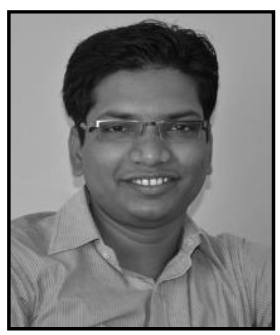

Ritesh Kumar Badhai was born in India on 1984. He received the Bachelor of engineering degree in Electronics and Communication Engineering from Rajiv Gandhi Proudyogiki Vishwavidyalaya, Bhopal, India in 2007 and Master of Engineering degree in Electronics and Communication Engineering from Birla Institute of Technology, Mesra, Ranchi, India in 2009. He currently is an Assistant Professor in the Department of Electronics and Communication Engineering, Birla Institute of Technology, Patna, Bihar, India and is also pursuing his Ph.D. degree.

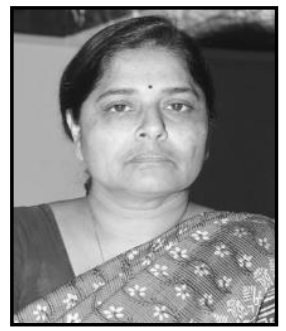

Nisha Gupta received the Bachelor's and Master's degrees in Electronics and Telecommunication and Electrical and Electronics engineering both from Birla Institute of Technology, Mesra, Ranchi, India and Ph.D. degree from the Indian Institute of Technology, Kharagpur, India. She was a post-doctoral fellow at University of Manitoba, Canada from 1997-1998 before joining the department of 
Electronics and Communication Engineering, Birla Institute of Technology in 1999 as a Reader. Currently, she is a Professor in the same department. She has authored and co-authored more than 100 technical journal articles and conference papers. Her research interests are Computational Electromagnetics, Antennas for Wireless Communication, AI techniques in Wireless and Mobile Communication and EMI/EMC. 
International Journal of Signal Processing, Image Processing and Pattern Recognition Vol.9, No.1 (2016) 